\title{
A Blind Adaptive Color Image Watermarking Scheme Based on Principal Component Analysis, Singular Value Decomposition and Human Visual System
}

\author{
Muhammad IMRAN, Bruce A. HARVEY \\ Department of Electrical \& Computer Engineering, College of Engineering \\ Florida State University, 2525 Pottsdamer St, Tallahassee, FL 32310, USA \\ mi14@my.fsu.edu, bharvey@fsu.edu
}

Submitted February 26, 2016 / Accepted July 11, 2017

\begin{abstract}
A blind adaptive color image watermarking scheme based on principal component analysis, singular value decomposition, and human visual system is proposed. The use of principal component analysis to decorrelate the three color channels of host image, improves the perceptual quality of watermarked image. Whereas, human visual system and fuzzy inference system helped to improve both imperceptibility and robustness by selecting adaptive scaling factor, so that, areas more prone to noise can be added with more information as compared to less prone areas. To achieve security, location of watermark embedding is kept secret and used as key at the time of watermark extraction, whereas, for capacity both singular values and vectors are involved in watermark embedding process. As a result, four contradictory requirements; imperceptibility, robustness, security and capacity are achieved as suggested by results. Both subjective and objective methods are acquired to examine the performance of proposed schemes. For subjective analysis the watermarked images and watermarks extracted from attacked watermarked images are shown. For objective analysis of proposed scheme in terms of imperceptibility, peak signal to noise ratio, structural similarity index, visual information fidelity and normalized color difference are used. Whereas, for objective analysis in terms of robustness, normalized correlation, bit error rate, normalized hamming distance and global authentication rate are used. Security is checked by using different keys to extract the watermark. The proposed schemes are compared with state-of-the-art watermarking techniques and found better performance as suggested by results.
\end{abstract}

\section{Keywords}

Image watermarking, principal component analysis, singular value decomposition, human visual system, imperceptibility, robustness

\section{Introduction}

The enormous usage and afford-ability of Internet across the world, has made it easy to access online literature in the form of pictures, audios, videos, books, etc. The data available online can be downloaded, and then can be redistributed its copies multiple times, without any distinguishable difference between original and copied material. This illegal distribution and copyright violation results in the form of millions of dollars loss [1]. To overcome this illicit distribution and copyright violation digital watermarking is proposed as a prominent solution [2-7].

Watermarking is the way of embedding some information (image, audio, strings) into another data (image, audio, video, pdf file). The embedded data can later be extracted to prove the ownership. If the data which is being protected by embedding information in it is image, then this type of watermarking is called image watermarking. The information which is being hidden is called watermark, and the image in which watermark is hidden is called host image, and the resultant image is known as watermarked image. In terms of information required at the time of watermark extraction, watermarking is divided into blind and non-blind [2]. In later case, original image is required (and may be key) at the time of watermark extraction, whereas in former watermarking type there is no need of original image for extraction of watermark [3]. Therefore, blind watermarking is considered to be more secure and convenient, hence in this paper blind watermarking schemes are proposed.

The watermarking scheme for gray scale [2], [4], [5] and for color images [1], [3], [6], [7] are available in literature. One additional challenge of color image watermarking is that the three channels $R, G$, and $B$ are highly correlated [3], [8], hence modification of one channel affects other channels severally and as a result the quality of whole image is compromised. This adverse effect can be avoided if Principal Component Analysis (PCA) [9] is used. In the proposed scheme PCA is used to decorrelate the three channels. In most cases either the same amount of information is em- 
bedded or modification in host image is made, same is case with [3], [6]. Whereas in actual there are some regions in image which are more tolerable to noise as compared to others. Therefore embedding more information in areas which are more prone to noise and less data into regions which are more susceptible to alterations. In this way both imperceptibility and robustness can be achieved simultaneously. For this purpose Human Visual System (HVS) and Fuzzy Inference System (FIS) are used to find adaptive scaling factor so that the amount of information is embedded according to the acceptability of host image.

In addition to perceptual quality of watermarked image and robustness, an other concern is that neither original nor false watermark should be extracted intentionally or unintentionally as was the case with [10-12]. Where, with the help of fake keys (singular vectors) a watermark different than the embedded watermark is extracted, which completely destroys the purpose of copyright protection. Therefore in designing proposed scheme, special attention is given to security and it is ensured that neither original nor false watermark is extracted as suggest by results in Sec. 5.3. The proposed scheme is discussed in detail in the following sections.

\section{Proposed Scheme}

In this paper two watermarking schemes are proposed. In proposed scheme 1, HVS and FIS are used together to find the areas where more information can be embedded, and regions where less data should be concealed. In this way two contradictory requirements of watermarking techniques; imperceptibility and robustness can be achieved. The perceptual quality of watermarked image is also improved due to the use of PCA. The third and utmost important quality is the security of watermarking scheme, which is given special attention in designing both the techniques. To achieve security, a different way of choosing elements for modifications from $U, S$ and $V$ are selected. In this way not only security but robustness and capacity are also improved as suggested by results in Sec. 5.2 and 5.3. In proposed scheme 2, HVS and FIS are not employed to find adaptive scaling factors, hence proposed scheme 1 is general case of proposed scheme 2 . Since the proposed schemes are mostly relying on SVD, therefore, techniques chosen for comparision are also based on SVD. Both schemes are compared with state-ofthe-art techniques [3], [6], [13], [14] and found that proposed schemes outperform. As HVS and FIS are used in this paper, therefore, they are discussed in following sections.

\subsection{Human Visual System}

Given an image $I$ of size $M \times N$, the luminance masking [15] $M_{L}$, is calculated as follows:

$$
M_{L}(x, y)=\max \left\{f_{1}(b g(x, y), \operatorname{mg}(x, y)), f_{2}(b g(x, y))\right\}
$$

where

$f_{1}(b g(x, y), m g(x, y))=m g(x, y) \alpha\{b g(x, y))+\beta(b g(x, y)\}$,

$f_{2}(b g(x, y))= \begin{cases}T_{o}\left(1-\left(\frac{b g(x, y)}{127}\right)^{0.5}\right)+3, & \operatorname{bg}(x, y) \leq 127, \\ \gamma(b g(x, y)-127)+3, & b g(x, y)>127,\end{cases}$

$\alpha(b g(x, y))=\left\{\begin{array}{l}0.0001 \operatorname{bg}(x, y)+0.115, \\ 1 \leq x \leq H, 1 \leq y \leq W,\end{array}\right.$

$\beta(\operatorname{bg}(x, y))=\lambda-0.01 \operatorname{bg}(x, y)$,

$f_{1}$ is the spatial masking function, $b g(x, y)$ is the background luminance, $\operatorname{mg}(x, y)$ is the maximum weighted average of luminance differences around the pixel at location $(x, y)$, $W=M / 2$ and $H=N / 2 . f_{2}, \alpha$ and $\beta$ are the background luminance dependent functions. The value of some other parameters are: $T_{0}=17, \gamma=3 / 128$, and $\lambda=1 / 2$. In order to know about the selection of parameter's values readers may refer [15]. $m g(x, y)$ and $b g(x, y)$ are calculated as follows:

$$
\begin{aligned}
m g(x, y) & =\max _{k=1,2,3,4}\left\{\left|\operatorname{grad}_{k}(x, y)\right|\right\} \\
\operatorname{grad}_{k}(x, y) & =\frac{1}{16} \sum_{i=1}^{5} \sum_{j=1}^{5} I(x-3+i, y-3+j) G_{k}(i, j) \\
b g(x, y) & =\frac{1}{32} \sum_{i=1}^{5} \sum_{j=1}^{5} I(x-3+i, y-3+j) B(i, j) .
\end{aligned}
$$

The values of $G_{1}, G_{2}, G_{3}, G_{4}$ and $B$ are shown below:

$$
\begin{gathered}
G_{1}=\left[\begin{array}{ccccc}
0 & 0 & 0 & 0 & 0 \\
1 & 3 & 8 & 3 & 1 \\
0 & 0 & 0 & 0 & 0 \\
-1 & -3 & -8 & -3 & -1 \\
0 & 0 & 0 & 0 & 0
\end{array}\right], G_{2}=\left[\begin{array}{ccccc}
0 & 0 & 1 & 0 & 0 \\
0 & 8 & 3 & 0 & 0 \\
1 & 3 & 0 & -3 & -1 \\
0 & 0 & -3 & -8 & 0 \\
0 & 0 & -1 & 0 & 0
\end{array}\right], \\
G_{3}=\left[\begin{array}{ccccc}
0 & 0 & 1 & 0 & 0 \\
0 & 0 & 3 & 8 & 0 \\
-1 & -3 & 0 & 3 & 1 \\
0 & -8 & -3 & 0 & 0 \\
0 & 0 & 1 & 0 & 0
\end{array}\right], G_{4}=\left[\begin{array}{ccccc}
0 & 1 & 0 & -1 & 0 \\
0 & 3 & 0 & -3 & 0 \\
0 & 8 & 0 & -8 & 0 \\
0 & 3 & 0 & -3 & 0 \\
0 & 1 & 0 & -1 & 0
\end{array}\right], \\
B=\left[\begin{array}{ccccc}
1 & 1 & 1 & 1 & 1 \\
1 & 2 & 2 & 2 & 1 \\
1 & 2 & 0 & 2 & 1 \\
1 & 2 & 2 & 2 & 1 \\
1 & 1 & 1 & 1 & 1
\end{array}\right] .
\end{gathered}
$$

Remark 1: Since, darkness is more tolerable (then brightness) to watermark [15], [16]. More alternation can be made into dark areas as compared to bright regions of image, in this way, imperceptibility and robustness can be achieved.

\subsection{Fuzzy Inference System}

Mamdani fuzzy inference system [17] is used to find the adaptive scaling factor $\alpha_{M_{L}}$ using luminance masking shown in (1). The membership functions for luminance masking is shown in Fig. 1. 


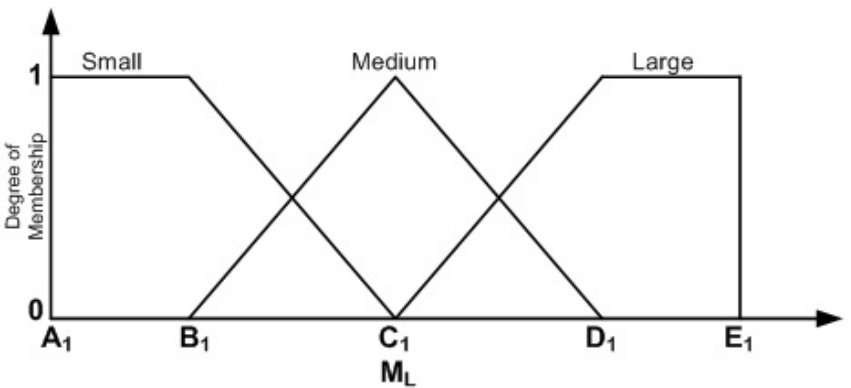

Fig. 1. Membership function for luminance masking.

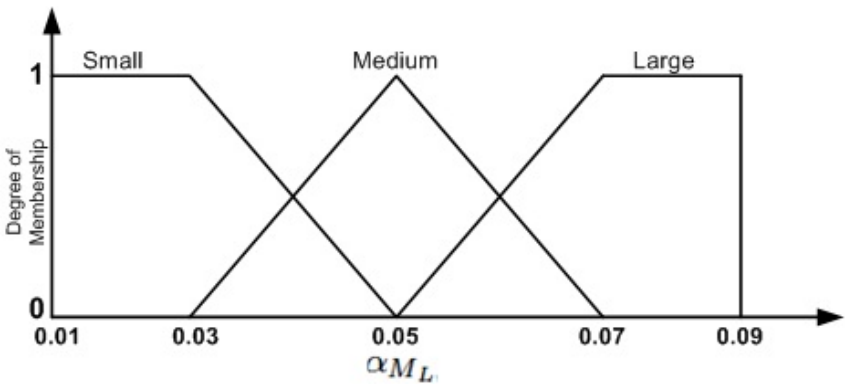

Fig. 2. Membership function for $\alpha_{M_{L}}$.

Where the values $A_{1}, B_{1}, C_{1}, D_{1}$ and $E_{1}$ are calculated as:

$$
\begin{aligned}
A_{1} & =\underset{x=1}{W} \min _{y=1}^{H}\left(M_{L}\right), \\
C_{1} & =\frac{1}{W H} \sum_{x=1}^{W} \sum_{y=1}^{H} M_{L}(x, y), \\
E_{1} & =\max _{x=1}^{W} \max _{y=1}^{H} M_{L}(x, y) .
\end{aligned}
$$

In order to chose $B_{1}$ and $D_{1}$, following condition must be satisfied

$$
C_{1}-B_{1}=D_{1}-C_{1} .
$$

The membership function for adaptive scaling factor $\alpha_{M_{L}}$ is shown in Fig. 2.

The rules used for calculating $\alpha_{M_{L}}$ are as follows:

$$
\begin{aligned}
& R u^{1}: \text { IF } M_{L} \text { is large, THEN } \alpha_{M_{L}} \text { is large, } \\
& R u^{2}: \text { IF } M_{L} \text { is medium, THEN } \alpha_{M_{L}} \text { is medium, } \\
& R u^{3}: \text { IF } M_{L} \text { is small, THEN } \alpha_{M_{L}} \text { is small. }
\end{aligned}
$$

The detailed procedure of watermark embedding and extraction are discussed in following sections.

\section{Proposed Scheme 1}

In proposed scheme 1, human visual system is used to find the areas of image which are more prone to alterations, and areas which are less prone to disturbances. The information of HVS is then used in FIS to find adaptive scaling factor for watermark embedding, so that the areas which are more prone to noise, can be embedded with more information as compared to areas which are less prone. In this way both; imperceptibility and robustness can be achieved as suggested by results in Sec. 5. The detailed procedure of watermark embedding and extraction are discussed in Sec. 3.1 and 3.2 respectively.

\subsection{Watermark Embedding}

1. Let the original image $I$ is decomposed into three channels $R, G$, and $B$

$$
\begin{aligned}
R= & {\left[\begin{array}{cccc}
r_{11} & r_{12} & \ldots & r_{1 N} \\
r_{21} & r_{22} & \ldots & r_{2 N} \\
\vdots & \vdots & \ddots & \vdots \\
r_{M 1} & r_{M 2} & \ldots & r_{M N}
\end{array}\right], } \\
G= & {\left[\begin{array}{cccc}
g_{11} & g_{12} & \ldots & g_{1 N} \\
g_{21} & g_{22} & \ldots & g_{2 N} \\
\vdots & \vdots & \ddots & \vdots \\
g_{M 1} & g_{M 2} & \ldots & g_{M N}
\end{array}\right], } \\
B= & {\left[\begin{array}{cccc}
b_{11} & b_{12} & \ldots & b_{1 N} \\
b_{21} & b_{22} & \ldots & b_{2 N} \\
\vdots & \vdots & \ddots & \vdots \\
b_{M 1} & b_{M 2} & \ldots & b_{M N}
\end{array}\right], }
\end{aligned}
$$

$M$ and $N$ defines the size of image.

2. Let a covariance matrix $C$ be computed as

$$
C=\frac{1}{M N}\left(A A^{\mathrm{T}}\right)=Q \wedge Q^{-1}
$$

where

$\mathbf{A}=$

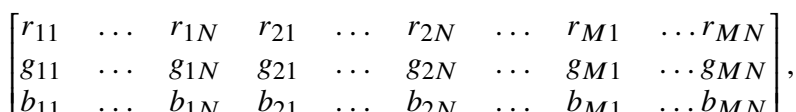

$Q=\left[\begin{array}{lll}q_{11} & q_{12} & q_{13} \\ q_{21} & q_{32} & q_{33} \\ q_{31} & q_{32} & q_{33}\end{array}\right], \quad \wedge=\left[\begin{array}{ccc}\lambda_{11} & 0 & 0 \\ 0 & \lambda_{22} & 0 \\ 0 & 0 & \lambda_{33}\end{array}\right]$,

$\lambda_{11} \geq \lambda_{22} \geq \lambda_{33}$ are eigenvalues in descending order.

3. The principal components [18] of covariance matrix $C$ are calculated as

$$
\begin{aligned}
& P=\left[\begin{array}{l}
P_{r} \\
P_{g} \\
P_{r}
\end{array}\right]=Q^{\mathrm{T}} A= \\
& =\left[\begin{array}{lllll}
p_{r_{11}} & \ldots & p_{r_{1 N}} & p_{r_{21}} & \ldots \\
p_{g_{11}} & \ldots & p_{g_{1 N}} & p_{g_{21}} & \ldots \\
p_{b_{11}} & \ldots & p_{b_{1 N}} & p_{b_{21}} & \ldots
\end{array}\right. \\
& \left.\ldots \begin{array}{cccccc}
\ldots & p_{r_{2 N}} & \ldots & p_{r_{M 1}} & \ldots & p_{r_{M N}} \\
\ldots & p_{g_{2 N}} & \ldots & p_{g_{M 1}} & \ldots & p_{g_{M N}}
\end{array}\right] . \\
& \left.\ldots \begin{array}{llllll}
\ldots & p_{b_{2 N}} & \ldots & p_{b_{M 1}} & \ldots & p_{b_{M N}}
\end{array}\right]
\end{aligned}
$$

Remark 2: Since $R, G$ and $B$ components are highly correlated [3], [7] and [8], modification in one channel causes alteration in other two channels, as a result imperceptibility is affected severely. However, this can be avoided if PCA [9] is used to decorrelate these $R, G$ and $B$ components. 
4. Let matrix $\mathbf{P}_{\mathbf{r n}}$ is formed from row vector $\mathbf{P}_{\mathbf{r}}$ of matrix $\mathbf{P}$ as shown below

$$
P_{r n}=\left[\begin{array}{cccc}
p_{r_{11}} & p_{r_{12}} & \ldots & p_{r_{1 N}} \\
p_{r_{21}} & p_{r_{22}} & \ldots & p_{r_{2 N}} \\
\vdots & \vdots & \ddots & \vdots \\
p_{r_{M 1}} & p_{r_{M 2}} & \cdots & p_{r_{M N}}
\end{array}\right] .
$$

Remark 3: Since $\mathbf{P}_{\mathbf{r n}}$ is composed of components from $\mathbf{P}_{\mathbf{r}}$ and contains most of the information [9], therefore it is chosen for watermark embedding.

5. Let $\mathbf{P}_{\text {rn }}$ is divided into non-overlapping blocks $\mathbb{A}_{\mathbf{1}}$, $\mathbb{A}_{\mathbf{2}}, \ldots \mathbb{A}_{\text {pq }}$. Where the size of each block is $4 \times 4$, $p \leq M / 4$ and $q \leq N / 4$.

6. Let each block is decomposed using SVD as follows

$$
\mathbb{A}_{i}=U_{i} S_{i} V_{i}^{\mathrm{T}}, \quad i=1,2, \ldots, p q
$$

where $S$ is a diagonal matrix containing singular values in descending order, whereas, $U$ and $V$ represent the left and right singular vectors respectively.

Remark 4: Small perturbation in image does not cause large variation in singular values and vice versa [5], [7].

Remark 5: Singular values contain intrinsic properties of image, whereas geometric information is maintained by corresponding singular vectors [5], [7].

7. Compute the covariance matrix of each block $\mathbb{A}_{i}$. Select the column number with lowest covariance (variance) value, and then location of two values with lowest covariance values within that selected column from covariance matrix of each block. In this way three numbers are selected for each block, and those selected locations will later be used as key at the time of watermark extraction. For instance $(f, e$ and $g$ ) are selected, where, ' $f$ ' represents the column number with lowest covariance value, ' $e$ ' and ' $g$ ' denoting the locations of two values with lowest covariance values from selected columns. These three numbers are used to select two numbers from $U$ and $V$ for modifications. For example in case of $(f, e$, and $g)$, tow numbers at location $(e, f)$ and $(g, f)$ from $U$, similarly two values from location $(f, e)$ and $(f, g)$ from $V$ are selected for watermark embedding. In addition to that singular values either at location $(e, e)$ or $(g, g)$ based on embedding bits are modified as shown in (7)-(10). It is shown in result in Sec. 5 that the procedure described above for choosing two numbers for modifications gives better results in terms of imperceptibility.

8. Let the luminance masking $M_{L_{i}}$ for each block $\mathbb{A}_{\mathbf{i}}$, where $i=1,2,3, \ldots, p q$ is calculated using (1).

9. The adaptive scaling factor $\alpha_{M L_{i}}(g, f), \alpha_{M L_{i}}(e, f)$, $\alpha_{M L_{i}}(f, g)$ and $\alpha_{M L_{i}}(f, e)$ of each block $\mathbb{A}_{\mathbf{i}}$ for values of selected positions are calculated by utilizing luminance masking values $M_{L_{i}}$ of block ' $i$ '.
10. Given a watermark $W$ of size $M / 4 \times N / 4$, the watermarking bits $W_{i}$ are embedded as described below in (7)-(10).

Case 1: If embedding bit is 1 i.e. $\left(W_{i}=1\right)$.

$$
\left.\begin{array}{l}
\overbrace{u_{w i(e, f)}=\operatorname{sgn}\left(u_{i(e, f)}\right) \times\left(\bar{U}_{i}+\left(\beta \alpha_{M L_{i}}(e, f)\right) / 2\right)}^{\text {if } e<g} \\
u_{w i(g, f)}=\operatorname{sgn}\left(u_{i(g, f)}\right) \times\left(\bar{U}_{i}-\left(\beta \alpha_{M L_{i}}(g, f)\right) / 2\right) \\
v_{w i(f, e)}=\operatorname{sgn}\left(v_{i(f, e)}\right) \times\left(\bar{V}_{i}+\left(\beta \alpha_{M L_{i}}(f, e)\right) / 2\right) \\
v_{w i(f, g)}=\operatorname{sgn}\left(v_{i(f, g)}\right) \times\left(\bar{V}_{i}-\left(\beta \alpha_{M L_{i}}(f, g)\right) / 2\right) \\
s_{w i(g, g)}=3 \times s_{i(e, e)}
\end{array}\right\}
$$

$$
\left.\begin{array}{l}
\overbrace{u_{w i(g, f)}=\operatorname{sgn}\left(u_{i(g, f)}\right) \times\left(\bar{U}_{i}+\left(\beta \alpha_{M L_{i}}(g, f)\right) / 2\right)}^{\text {If } e>g} \\
u_{w i(e, f)}=\operatorname{sgn}\left(u_{i(e, f)}\right) \times\left(\bar{U}_{i}-\left(\beta \alpha_{M L_{i}}(e, f)\right) / 2\right) \\
v_{w i(f, g)}=\operatorname{sgn}\left(v_{i(f, g)}\right) \times\left(\bar{V}_{i}+\left(\beta \alpha_{M L_{i}}(f, g)\right) / 2\right) \\
v_{w i(f, e)}=\operatorname{sgn}\left(v_{i(f, e)}\right) \times\left(\bar{V}_{i}-\left(\beta \alpha_{M L_{i}}(f, e)\right) / 2\right) \\
s_{w i(e, e)}=3 \times s_{i(g, g)}
\end{array}\right\}
$$

Case 2: If embedding bit is 0 i.e. $\left(W_{i}=0\right)$.

$$
\left.\begin{array}{l}
\overbrace{u_{w i(e, f)}=\operatorname{sgn}\left(u_{i(e, f)}\right) \times\left(\bar{U}_{i}-\left(\beta \alpha_{M L_{i}}(e, f)\right) / 2\right)}^{\text {If } e<g} \\
u_{w i(g, f)}=\operatorname{sgn}\left(u_{i(g, f)}\right) \times\left(\bar{U}_{i}+\left(\beta \alpha_{M L_{i}}(g, f)\right) / 2\right) \\
v_{w i(f, e)}=\operatorname{sgn}\left(v_{i(f, e)}\right) \times\left(\bar{V}_{i}-\left(\beta \alpha_{M L_{i}}(f, e)\right) / 2\right) \\
v_{w i(f, g)}=\operatorname{sgn}\left(v_{i(f, g)}\right) \times\left(\bar{V}_{i}+\left(\beta \alpha_{M L_{i}}(f, g)\right) / 2\right) \\
s_{w i(g, g)}=3 \times s_{i(e, e)}
\end{array}\right\}
$$

$$
\left.\begin{array}{l}
\overbrace{u_{w i(g, f)}=\operatorname{sgn}\left(u_{i(g, f)}\right) \times\left(\bar{U}_{i}-\left(\beta \alpha_{M L_{i}}(g, f)\right) / 2\right)} \\
u_{w i(e, f)}=\operatorname{sgn}\left(u_{i(e, f)}\right) \times\left(\bar{U}_{i}+\left(\beta \alpha_{M L_{i}}(e, f)\right) / / 2\right) \\
v_{w i(f, g)}=\operatorname{sgn}\left(v_{i(f, g)}\right) \times\left(\bar{V}_{i}-\left(\beta \alpha_{M L_{i}}(f, g)\right) / / 2\right) \\
v_{w i(f, e)}=\operatorname{sgn}\left(v_{i(f, e)}\right) \times\left(\bar{V}_{i}+\left(\beta \alpha_{M L_{i}}(f, e)\right) / / 2\right) \\
s_{w i(e, e)}=3 \times s_{i(g, g)}
\end{array}\right\}
$$

where

$$
\operatorname{sgn}(x)= \begin{cases}-1, & \text { if } x<0 \\ 0, & \text { if } x=0 \\ 1, & \text { if } x>0\end{cases}
$$

$$
\bar{U}_{i}=\frac{\left|u_{i(e, f)}+u_{i(g, f)}\right|}{2}, \quad \bar{V}_{i}=\frac{\left|v_{i(f, e)}+v_{i(f, g)}\right|}{2} .
$$

$W_{i}$ represents the watermark bit, where $1 \leq i \leq$ $M N / 16$. In (7)-(10), $\beta$ represents the threshold defin-

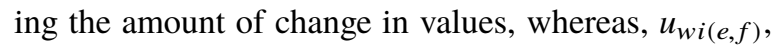
$u_{w i(g, f)}, v_{w i(f, e)}, v_{w i(f, g)}, s_{w i(e, e)}$, and $s_{w i(g, g)}$ represents the modified (watermark added) values at locations $(e, f),(g, f),(f, e),(f, g),(e, e)$ and $(g, g)$ respectively for block $i$. For (7) and (9) the key would be 
$\mathbb{K}_{i}=\{f, e, g\}$ whereas in case of (8) and (10) key would be $\mathbb{K}_{i}=\{f, g, e\}$ for block $i$.

Remark 6: Modification in columns of $U$ as compared to rows of $U$ are less visible, likewise, in $V$ the modification in rows are less visible as compared to changes in columns in $V$ [4].

11. Let the modified blocks are formed as follows

$$
\mathbb{A}_{w i}=U_{w i} S_{w i} V_{w i}^{\mathrm{T}}, \quad i=1,2, \ldots p q
$$

where, the subscript ' $w$ ' is representing the modified (or watermark added) blocks.

12. The modified blocks $\mathbb{A}_{w 1}, \mathbb{A}_{w 2}, \ldots, \mathbb{A}_{w p q}$ are combined to form modified first principal component $P_{r n w}$, where

$$
P_{r n w}=\left[\begin{array}{cccc}
P_{r w_{11}} & P_{r w_{12}} & \ldots & P_{r w_{1 N}} \\
P_{r w_{21}} & P_{r w_{22}} & \ldots & P_{r w_{2 N}} \\
\vdots & \vdots & \ddots & \vdots \\
P_{r w_{M 1}} & P_{r w_{M 2}} & \ldots & P_{r w_{M N}}
\end{array}\right]
$$

13. The modified principal components are obtained as

$$
P_{w}=\left[\begin{array}{c}
P_{r w} \\
P_{g} \\
P_{b}
\end{array}\right]
$$

where $\mathbf{P}_{\text {rw }}$ is a row vector, obtained from $\mathbf{P}_{\text {rnw }}$.

14. The matrix $A_{w}$ is obtained as

$$
\begin{aligned}
& A_{w}=Q P_{w}= \\
& =\left[\begin{array}{ccccc}
r w_{11} & \ldots & r w_{1 N} & r w_{21} & \ldots \\
g_{11} & \ldots & g_{1 N} & g_{21} & \ldots \\
b_{11} & \ldots & b_{1 N} & b_{21} & \ldots
\end{array}\right. \\
& \left.\begin{array}{ccccc}
\ldots & r w_{2 N} & \ldots & r w_{M 1} & \ldots r w_{M N} \\
\ldots & g_{2 N} & \ldots & g_{M 1} & \ldots g_{M N} \\
& b_{2 N} & \ldots & b_{M 1} & \ldots b_{M N}
\end{array}\right]
\end{aligned}
$$

15. Finally, $R_{W}, G$ and $B$ channels are combined to form the watermarked image $I_{w}$, where

$$
R_{W}=\left[\begin{array}{cccc}
r w_{11} & r w_{11} & \ldots & r w_{1 N} \\
r w_{21} & r w_{22} & \ldots & r w_{2 N} \\
\vdots & \vdots & \ddots & \vdots \\
r w_{M 1} & r w_{M 2} & \ldots & r w_{M N}
\end{array}\right] .
$$

\subsection{Watermark Extraction}

1. Let received watermarked image $\hat{I}_{w}$ be decomposed into its components $\hat{R}_{w}, \hat{G}$, and $\hat{B}$, where

$$
\hat{R}_{w}=\left[\begin{array}{cccc}
r \hat{w}_{11} & r \hat{w}_{12} & \ldots & r \hat{w}_{1 N} \\
r \hat{w}_{21} & r \hat{w}_{22} & \ldots & r \hat{w}_{2 N} \\
\vdots & \vdots & \ddots & \vdots \\
r \hat{w}_{M 1} & r \hat{w}_{M 2} & \ldots & r \hat{w}_{M N}
\end{array}\right]
$$

$$
\begin{array}{r}
\hat{G}=\left[\begin{array}{cccc}
\hat{g}_{11} & \hat{g}_{12} & \ldots & \hat{g}_{1 N} \\
\hat{g}_{21} & \hat{g}_{22} & \ldots & \hat{g}_{2 N} \\
\vdots & \vdots & \ddots & \vdots \\
\hat{g}_{M 1} & \hat{g}_{M 2} & \ldots & \hat{g}_{M N}
\end{array}\right], \\
\hat{B}=\left[\begin{array}{cccc}
\hat{b}_{11} & \hat{b}_{12} & \ldots & \hat{b}_{1 N} \\
\hat{b}_{21} & \hat{b}_{22} & \ldots & \hat{b}_{2 N} \\
\vdots & \vdots & \ddots & \vdots \\
\hat{b}_{M 1} & \hat{b}_{M 2} & \ldots & \hat{b}_{M N}
\end{array}\right] .
\end{array}
$$

2. Let a covariance matrix $\hat{C}$ be computed as

$$
\hat{C}=\frac{1}{M N}\left(\hat{A} \hat{A}^{\mathrm{T}}\right)=\hat{Q} \hat{\wedge} \hat{Q}^{-1}
$$

where

$$
\begin{gathered}
\hat{A}=\left[\begin{array}{ccccc}
r \hat{w}_{11} & \ldots & r \hat{w}_{1 N} & r \hat{w}_{21} & \ldots \\
\hat{g}_{11} & \ldots & \hat{g}_{1 N} & \hat{g}_{21} & \ldots \\
\hat{b}_{11} & \ldots & \hat{b}_{1 N} & \hat{b}_{21} & \ldots \\
\ldots & r \hat{w}_{2 N} & \ldots & r \hat{w}_{M 1} & \ldots r \hat{w}_{M N} \\
\ldots & \hat{g}_{2 N} & \ldots & \hat{g}_{M 1} & \ldots \hat{g}_{M N} \\
\ldots & \hat{b}_{2 N} & \ldots & \hat{b}_{M 1} & \ldots \hat{b}_{M N}
\end{array}\right] . \\
\hat{Q}=\left[\begin{array}{lll}
\hat{q}_{11} & \hat{q}_{12} & \hat{q}_{13} \\
\hat{q}_{21} & \hat{q}_{32} & \hat{q}_{33} \\
\hat{q}_{31} & \hat{q}_{32} & \hat{q}_{33}
\end{array}\right], \quad \hat{\wedge}=\left[\begin{array}{ccc}
\hat{\lambda}_{11} & 0 & 0 \\
0 & \hat{\lambda}_{22} & 0 \\
0 & 0 & \hat{\lambda}_{33}
\end{array}\right],
\end{gathered}
$$

$\hat{\lambda}_{11} \geq \hat{\lambda}_{22} \geq \hat{\lambda}_{33}$ are eigenvalues in descending order.

3. The principal components of covariance matrix $\hat{C}$ are calculated as

$$
\begin{aligned}
& \hat{P}_{w}=\left[\begin{array}{c}
\hat{P}_{r w} \\
\hat{P}_{g} \\
\hat{P}_{b}
\end{array}\right]=\hat{Q}^{\mathrm{T}} \hat{A}= \\
& =\left[\begin{array}{ccccc}
\hat{P}_{r w_{11}} & \ldots & \hat{P}_{r w_{1 N}} & \hat{P}_{r w_{21}} & \ldots \\
\hat{P}_{g_{11}} & \ldots & \hat{P}_{g_{1 N}} & \hat{P}_{g_{21}} & \ldots \\
\hat{P}_{b_{11}} & \ldots & \hat{P}_{b_{1 N}} & \hat{P}_{b_{21}} & \ldots
\end{array}\right. \\
& \left.\begin{array}{cccccc}
\ldots & \hat{P}_{r w_{2 N}} & \ldots & \hat{P}_{r w_{M 1}} & \ldots & \hat{P}_{r w_{M N}} \\
\ldots & \hat{P}_{g_{2 N}} & \ldots & \hat{P}_{g_{M 1}} & \ldots & \hat{P}_{g_{M N}} \\
\ldots & \hat{P}_{b_{2 N}} & \ldots & \hat{P}_{b_{M 1}} & \ldots & \hat{P}_{b_{M N}}
\end{array}\right] .
\end{aligned}
$$

4. Let matrix $\hat{\mathbf{P}}_{\text {rnw }}$ is obtained by converting row vector $\mathbf{P}_{\text {rw }}$ into a matrix of size $M \times N$ as shown below

$$
\hat{P}_{r n w}=\left[\begin{array}{cccc}
\hat{P}_{r w_{11}} & \hat{P}_{r w_{12}} & \ldots & \hat{P}_{r w_{1 N}} \\
\hat{P}_{r w_{21}} & \hat{P}_{r w_{22}} & \ldots & \hat{P}_{r w_{2 N}} \\
\vdots & \vdots & \ddots & \vdots \\
\hat{P}_{r w_{M 1}} & \hat{P}_{r w_{M 2}} & \ldots & \hat{P}_{r w_{M N}}
\end{array}\right]
$$

5. The $\hat{\mathbf{P}}_{\text {rnw }}$ is divided into non-overlapping blocks $\hat{\mathbb{A}}_{w 1}, \hat{\mathbb{A}}_{w 2}, \ldots \hat{\mathbb{A}}_{w p q}$, each of size $4 \times 4$. Using SVD, each block is decomposed as follows:

$$
\hat{A}_{w i}=\hat{U}_{w i} \hat{S}_{w i} \hat{V}_{w i}^{\mathrm{T}}, \quad i=1,2, \ldots, p q .
$$


6. Based on keys: $\mathbb{K}_{i}=\left\{k_{1}, k_{2}, k_{3}\right\}$, the watermarking bits are extracted as follows:

$$
\begin{aligned}
& \xi= \begin{cases}1, & \text { if } \quad \hat{u}_{w i\left(k_{2}, K_{1}\right)} \leq \hat{u}_{w i\left(k_{3}, K_{1}\right)}, \\
0, & \text { otherwise. }\end{cases} \\
& \zeta= \begin{cases}1, & \text { if } \quad \hat{v}_{w i\left(k_{1}, K_{2}\right)} \leq \hat{v}_{w i\left(k_{1}, K_{3}\right)}, \\
0, & \text { otherwise. }\end{cases} \\
& \psi= \begin{cases}1, & \text { if } \quad \hat{s}_{w i\left(k_{2}, K_{2}\right)} \leq \hat{s}_{w i\left(k_{3}, K_{3}\right)}, \\
0, & \text { otherwise. }\end{cases}
\end{aligned}
$$

Once three values $\xi, \zeta$, and $\psi$ are calculated for each block, then watermarking bits are extracted as follows.

$$
\hat{W}_{i}= \begin{cases}\psi, & \text { if } \quad(\xi=\psi) \vee(\zeta=\psi), \\ \vartheta, & \text { otherwise }\end{cases}
$$

where

$$
\vartheta=\operatorname{Mode}\{\xi, \psi, \zeta\}
$$

\section{Proposed Scheme 2}

The only difference in proposed scheme 1 and 2 is that, in scheme 2 neither HVS nor FIS used to find adaptive factor $\alpha_{M_{L}}$ for watermark embedding. Instead only constant scaling factor $\beta$ having values from 0.1 to 0.9 with a step size of 0.2 is used. The watermark embedding and extraction procedure are described in following sections.

\subsection{Watermark Embedding}

Eliminating step 8 and step 9, and the terms $\alpha_{M L_{i}}(g, f)$, $\alpha_{M L_{i}}(e, f), \alpha_{M L_{i}}(f, g)$ and $\alpha_{M L_{i}}(f, e)$ from (7)-(10) of Sec. 3.1 will reduce to watermark embedding procedure of proposed scheme 2 .

\subsection{Watermark Extraction}

The watermark extraction procedure of proposed scheme 2 is same as of proposed scheme 1 which is described in Sec. 3.2.

\section{Experimental Results}

In order to test the performance of proposed schemes a number of experiments were performed. For this purpose six different images shown in Fig. 3, each of size $512 \times 512$ were used as host images. Whereas for watermark a binary image shown in Fig. 4(a) of size $64 \times 64$ was used. The performance of proposed schemes is evaluated in terms of imperceptibility, robustness, security and capacity, which are discussed in following sections.

\subsection{Imperceptibility}

To end user the original and watermarked image should look similar, in other words, there should be no visible difference between original and watermarked images [3], this is referred as imperceptibility. For qualitative analysis watermarked images are shown in Fig. 3, whereas, for quantitative evaluation Peak-Signal-to-Noise-Ration (PSNR) [7] shown in (18) is used to measure imperceptibility. The PSNR for proposed scheme 1 for different images and for distinct constant scaling factor is shown in Tab. 1. The use of constant scaling factor here is only for reference, otherwise, proposed scheme 1 performs well without constant scaling factor. The watermarked images are shown for proposed scheme 1, however, the watermarked images obtained from proposed scheme 2 also have good perceptual quality.

$$
\operatorname{PSNR}(\mathrm{dB})=10 \log _{10}\left(\frac{G^{2}}{H}\right)
$$

where

$$
G=\max \{I(m, n): 1 \leq m \leq M, 1 \leq n \leq N\},
$$

$$
H=\frac{1}{M \times N} \sum_{m=1}^{M} \sum_{n=1}^{N}\left(I(m, n)-I_{w}(m, n)\right)^{2} .
$$

\begin{tabular}{|l|l|l|l|l|l|}
\hline \multirow{2}{*}{ Images } & \multicolumn{5}{|c|}{ Constant Scaling Factor $(\beta)$} \\
\cline { 2 - 6 } & $\mathbf{0 . 1}$ & $\mathbf{0 . 3}$ & $\mathbf{0 . 5}$ & $\mathbf{0 . 7}$ & $\mathbf{0 . 9}$ \\
\hline Lena & 57.2311 & 56.5784 & 55.9255 & 55.2696 & 54.6518 \\
\hline Baboon & 46.7905 & 46.5187 & 46.2263 & 45.9465 & 45.6602 \\
\hline Autumn & 55.6855 & 53.8721 & 51.8756 & 50.1673 & 48.5929 \\
\hline Airplane & 55.0401 & 54.4737 & 53.9122 & 53.3167 & 52.7232 \\
\hline Peppers & 59.5511 & 58.4239 & 57.2720 & 56.1712 & 55.0926 \\
\hline Crane & 54.5905 & 54.1370 & 53.6779 & 53.2249 & 52.7693 \\
\hline
\end{tabular}

Tab. 1. PSNR for different values of scaling factors (Proposed scheme 1).

The proposed schemes are compared with state-of-theart schemes in terms of PSNR as shown in Tab. 2. It is clearly visible that both proposed schemes performs well with the compared techniques in terms of PSNR. However, the proposed scheme 1 outperforms proposed scheme 2 in terms of imperceptibility, and that is due to the use of HVS and FIS to find adaptive scaling factors.

\begin{tabular}{|l|c|c|c|c|c|c|}
\hline \multirow{2}{*}{ Images } & \multicolumn{6}{|c|}{ For Constant Scaling Factor $\beta=\mathbf{0 . 5}$} \\
\cline { 2 - 7 } & \multicolumn{2}{|c|}{ Proposed } & \multicolumn{4}{c|}{ Presented in } \\
\cline { 2 - 7 } & 1 & 2 & {$[6]$} & {$[3]$} & {$[13]$} & {$[14]$} \\
\hline Lena & 55.9255 & 42.0455 & 38.7070 & 36.1329 & 38.9620 & 34.3263 \\
\hline Baboon & 46.2263 & 37.3587 & 30.2003 & 27.5918 & 33.0635 & 27.3159 \\
\hline Autumn & 51.8756 & 41.7374 & 37.9715 & 35.0159 & 36.0157 & 33.6153 \\
\hline Airplane & 53.9122 & 39.9456 & 38.8983 & 35.4927 & 37.3391 & 34.7828 \\
\hline Peppers & 57.2720 & 39.6113 & 38.6906 & 34.2894 & 38.8031 & 33.2607 \\
\hline Crane & 53.6779 & 45.7264 & 44.9576 & 38.5728 & 40.0731 & 37.0299 \\
\hline
\end{tabular}

Tab. 2. PSNR for different techniques.

Generally the quality of watermarked images can either be evaluated qualitatively or qualitatively. For qualitative evaluation of proposed scheme both original and watermarked images are shown in Fig. 3. For quantitative measurement, PSNR shown in (18) is used, the results and comparision are shown in Tab. 1 and Tab. 2 respectively. However, PSNR is not only the metric to measure the quality of watermarked images, Structural Similarity Index (SSIM), Visual 


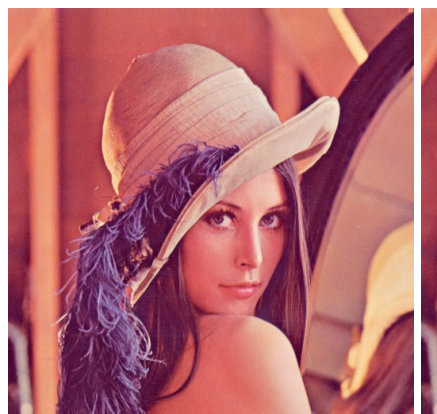

(a)

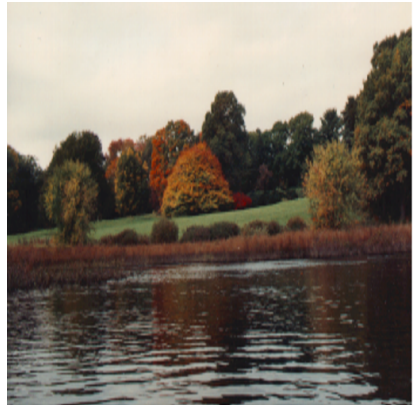

(e)

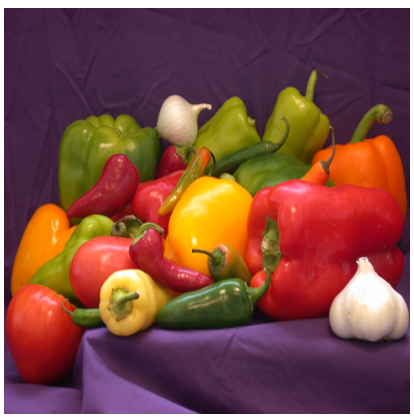

(i)

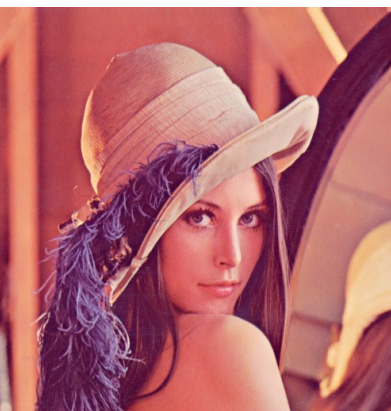

(b)

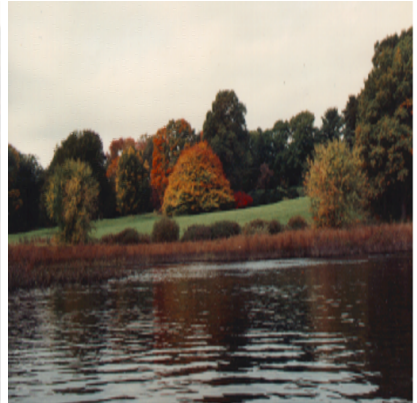

(f)

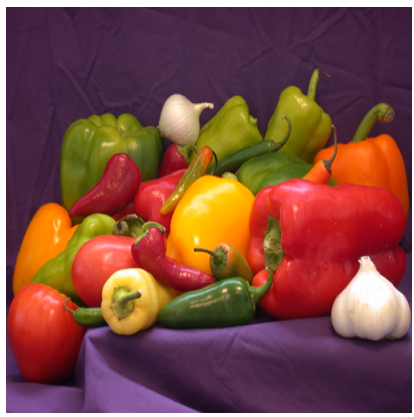

(j)

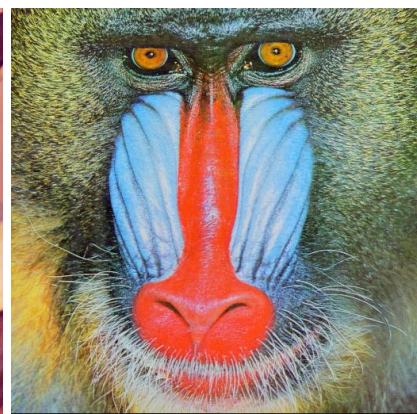

(c)

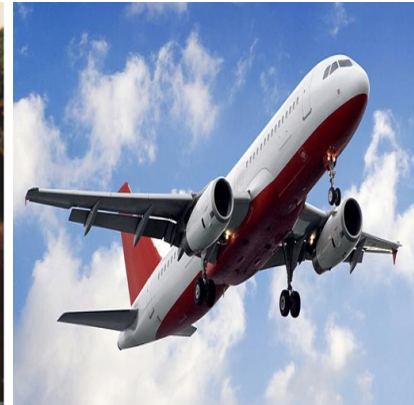

(g)

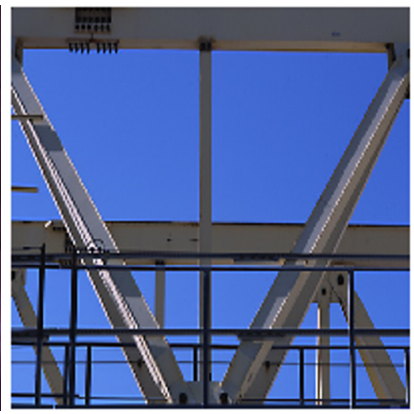

(k)

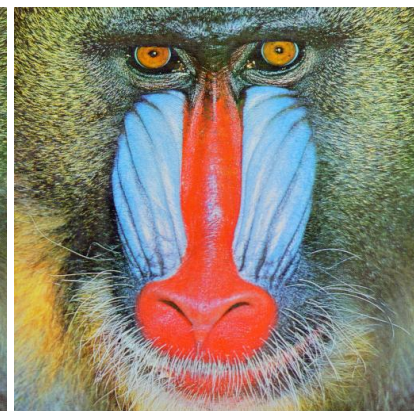

(d)

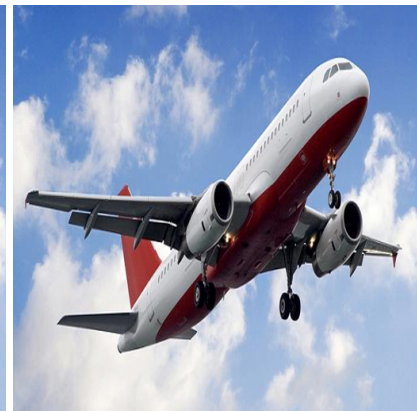

(h)

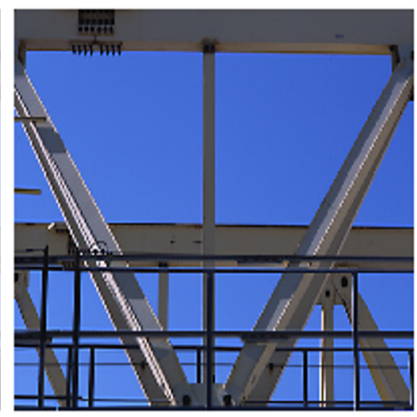

(1)

Fig. 3. Host and watermarked images $(512 \times 512)$ for proposed scheme $1(\beta=0.5)$ (a). Lena (original), (b). Lena (watermarked) (c). Baboon (original) (d). Baboon (watermarked) (e). Autumn (original) (f). Autumn (watermarked) (g). Airplane (original) (h). Airplane (watermarked) (i). Peppers (original) (j). Peppers (watermarked) (k). Crane (original) (l). Crane (watermarked).

Information Fidelity (VIF) and Normalized Color Difference (NCD) also used to examine the quality of watermarked images, quantitatively. Therefore, in this paper in addition to PSNR, SSIM, VIF and NCD are also utilized for evaluation [3]. SSIM [19] utilizes luminance $(\mathcal{L})$, contrast $(C)$ and structural information $(\mathcal{S})$ to find the distortion introduced in watermarked images. Given original $I$ and watermarked $I_{w}$ images, the SSIM can be calculated using (19).

$$
\operatorname{SSIM}(I, I w)=\mathcal{L}\left(I, I_{w}\right)^{\gamma_{\mathcal{L}}} \times \mathcal{S}\left(I, I_{w}\right)^{\gamma_{\mathcal{S}}} \times C\left(I, I_{w}\right)^{\gamma_{C}}
$$

where $\gamma_{\mathcal{L}}>0, \gamma_{\mathcal{L}}>0$ and $\gamma_{\mathcal{L}}>0$, are constant used to describe the dependency of each component. For equal contribution of $\mathcal{L}, \mathcal{S}$ and $C$ in the calculation of SSIM, $\gamma_{\mathcal{L}}=\gamma_{\mathcal{S}}=\gamma_{C}=1$ are set equal to 1 . The $\mathcal{L}, C$ and $\mathcal{S}$ defined in (19) are calculated as follows.

$$
\begin{aligned}
& \mathcal{L}\left(I, I_{w}\right)=\frac{2 \mu_{I} \mu_{I_{w}}+C_{1}}{\mu_{I}^{2}+\mu_{I_{w}}^{2}+C_{1}}, C\left(I, I_{w}\right)=\frac{2 \rho_{I} \rho_{I_{w}}+C_{2}}{\rho_{I}^{2}+\rho_{I_{w}}^{2}+C_{2}}, \\
& \mathcal{S}\left(I, I_{w}\right)=\frac{\rho_{I I_{w}}+C_{3}}{\rho_{I}^{2} \rho_{I_{w}}^{2}+C_{3}}
\end{aligned}
$$

where $\mu_{I}, \mu_{I_{w}}, \rho_{I}$ and $\rho_{I_{w}}$ denotes the mean and covariance of host and watermarked images respectively. $C_{1}, C_{2}$ and $C_{3}$ are small constants used to avoid the situations where the sum of means or covariances can be zero. Using (20) and setting $C_{3}=C_{2} / 2$ in (19), will result in the form of equation shown below

$$
S S I M=\frac{\left(2 \mu_{I} \mu_{I_{w}}+C_{1}\right) \times\left(2 \rho_{I} \rho_{I_{w}}+C_{2}\right)}{\left(2 \mu_{I}^{2}+\mu_{I_{w}}^{2}+C_{1}\right) \times\left(2 \rho_{I}^{2}+\rho_{I_{w}}^{2}+C_{2}\right)} .
$$




\begin{tabular}{|c|c|c|c|c|c|c|}
\hline \multirow{2}{*}{ Images } & \multicolumn{6}{|c|}{ For Constant Scaling Factor $\beta=\mathbf{0 . 5}$} \\
\cline { 2 - 7 } & \multicolumn{2}{|c|}{ Proposed } & \multicolumn{4}{c|}{ Presented in } \\
\cline { 2 - 7 } & 1 & 2 & {$[6]$} & {$[3]$} & {$[13]$} & {$[14]$} \\
\hline Lena & 0.9580 & 0.9088 & 0.8366 & 0.7810 & 0.8422 & 0.6881 \\
\hline Baboon & 0.9118 & 0.8796 & 0.7911 & 0.7228 & 0.8661 & 0.5972 \\
\hline Autumn & 0.9439 & 0.9279 & 0.8442 & 0.7785 & 0.8007 & 0.7266 \\
\hline Airplan & 0.9428 & 0.8921 & 0.8687 & 0.7926 & 0.8338 & 0.7383 \\
\hline Peppers & 0.9653 & 0.9130 & 0.8918 & 0.7904 & 0.8944 & 0.6775 \\
\hline Crane & 0.9259 & 0.9033 & 0.8574 & 0.7356 & 0.7642 & 0.6797 \\
\hline
\end{tabular}

Tab. 3. SSIM for different techniques.

The comparision of proposed schemes with existing techniques in terms of SSIM shown in Tab. 3, clearly demonstrates the improvement of proposed schemes over present watermarking techniques.

VIF [20] introduced in 2006 is also used to asses the quality of images. In this paper VIF shown in (22), is also used to examine the quality of watermarked images with respect to original host images

$$
V I F=\frac{\sum_{j \in \text { channels }} \mathbb{I}\left(\vec{C}^{N, j}, \vec{I}^{N, j} \mid s^{N, j}\right)}{\sum_{j \in \text { channels }} \mathbb{I}\left(\vec{C}^{N, j},{\overrightarrow{I_{w}}}^{N, j} \mid s^{N, j}\right)}
$$

where $\mathbb{I}\left(\vec{C}^{N, j}, \vec{I}^{N, j} \mid s\right)$ and $\mathbb{I}\left(\vec{C}^{N, j}, \vec{I}^{N, j} \mid s\right)$ represent the information that a brain can extract from images using HVS of original and watermarked images respectively, $\vec{C}^{N, j}$ represents the $N$ elements of random field $C_{j}$ for $j-t h$ sub-band. For detailed description of parameters and calculation of information $\mathbb{I}$ from host and watermarked images, [20] can be referred.

The comparison in terms of VIF of proposed schemes with that of state-of-the-art techniques is shown in Tab. 4. The better visual quality of watermarked images obtained from proposed schemes is clearly visible from the results shown in Tab. 4.

Finally, NCD [3] shown in (23), is also used to evaluate the quality of watermarked images.

$$
N C D=\frac{\sum_{i=1}^{M} \sum_{j=1}^{N} \sqrt{\left(\mathcal{L}(i, j)-\mathcal{L}_{w}(i, j)\right)^{2}+\left(a(i, j)-a_{w}(i, j)\right)^{2}+\left(b(i, j)-b_{w}(i, j)\right)^{2}}}{\sum_{i=1}^{M} \sum_{j=1}^{N} \sqrt{(\mathcal{L}(i, j))^{2}+(a(i, j))^{2}+(b(i, j))^{2}}}
$$

where $\mathcal{L}$ represents the luminance, $a$ and $b$ denote the chrominance. It should be noted that in order to calculate NCD, the RGB color model must be converted to $L a b$ color space. The performance of proposed scheme is calculated in terms of NCD and compared with existing schemes as shown in Tab. 5.

\begin{tabular}{|c|c|c|c|c|c|c|}
\hline \multirow{2}{*}{ Images } & \multicolumn{6}{|c|}{ For Constant Scaling Factor $\beta=\mathbf{0 . 5}$} \\
\cline { 2 - 7 } & \multicolumn{2}{|c|}{ Proposed } & \multicolumn{4}{c|}{ Presented in } \\
\cline { 2 - 7 } & 1 & 2 & {$[6]$} & {$[3]$} & {$[13]$} & {$[14]$} \\
\hline Lena & 0.8959 & 0.8423 & 0.7954 & 0.7425 & 0.8006 & 0.7053 \\
\hline Baboon & 0.8584 & 0.8154 & 0.7592 & 0.6936 & 0.7811 & 0.6866 \\
\hline Autumn & 0.8764 & 0.8282 & 0.7535 & 0.6948 & 0.7146 & 0.6670 \\
\hline Airplane & 0.8886 & 0.8343 & 0.8124 & 0.7413 & 0.7799 & 0.7265 \\
\hline Peppers & 0.9138 & 0.8779 & 0.8575 & 0.7600 & 0.8600 & 0.7372 \\
\hline Crane & 0.8805 & 0.8548 & 0.8404 & 0.7210 & 0.7490 & 0.6921 \\
\hline
\end{tabular}

Tab. 4. VIF for different techniques.

\begin{tabular}{|c|c|c|c|c|c|c|}
\hline \multirow{2}{*}{ Images } & \multicolumn{6}{|c|}{ For Constant Scaling Factor $\beta=\mathbf{0 . 5}$} \\
\cline { 2 - 7 } & \multicolumn{2}{|c|}{ Proposed } & \multicolumn{4}{c|}{ Presented in } \\
\cline { 2 - 7 } & 1 & 2 & {$[6]$} & {$[3]$} & {$[13]$} & {$[14]$} \\
\hline Lena & 0.0233 & 0.0408 & 0.0784 & 0.1516 & 0.0619 & 0.1164 \\
\hline Baboon & 0.0716 & 0.1295 & 0.2342 & 0.4482 & 0.1889 & 0.3450 \\
\hline Autumn & 0.0579 & 0.1045 & 0.1996 & 0.3837 & 0.2110 & 0.4079 \\
\hline Airplane & 0.0209 & 0.0364 & 0.0718 & 0.1373 & 0.0671 & 0.1296 \\
\hline Peppers & 0.0448 & 0.0758 & 0.1498 & 0.2826 & 0.0972 & 0.1805 \\
\hline Crane & 0.0268 & 0.0496 & 0.0984 & 0.1828 & 0.0871 & 0.1676 \\
\hline
\end{tabular}

Tab. 5. NCD for different techniques.

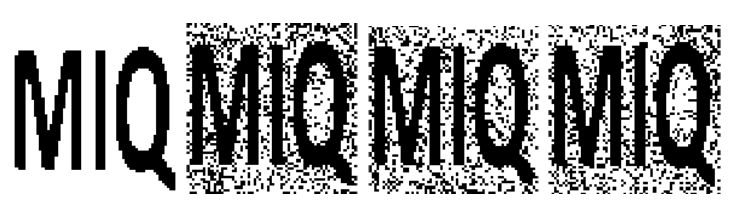

(a)

(b)

(c)

(d)

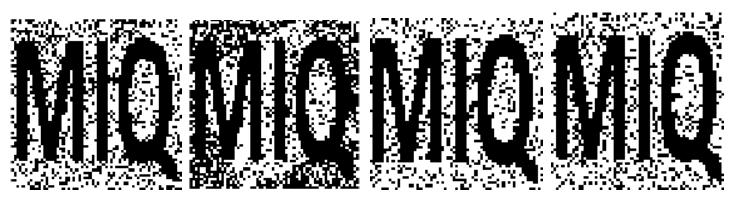

(e)

(f)

(g)

(h)

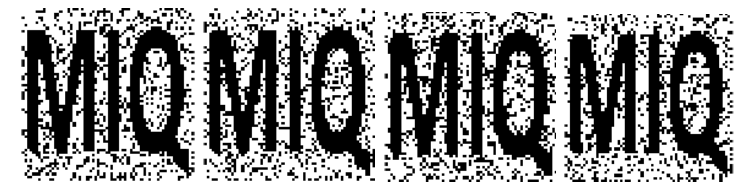

(i)

(j)

(k)

(1)

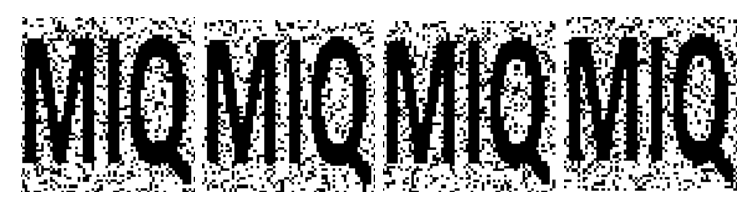

(m)

(n)

(o)

(p)

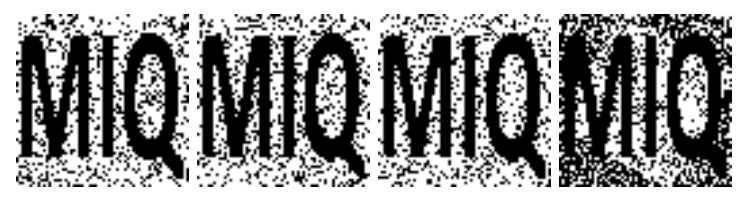

(q)

(r)

(s)

(t)

Fig. 4. Extracted watermarks from attacked watermarked images (proposed scheme $1(\beta=0.5)$ ): (a) Original watermark (b) RO, (c) TR, (d) XSH, (e) YSH, (f) AFT, (g). SC, (h) CR, (i). GN, (j) S\&P (k) SN, (l) MB, (m) SB, (n) $\mathrm{AF}$, (o) HE, (p) JQ (q) JQ+AF, (r) JQ+GN, (s) JQ+SC (t) $\mathrm{RO}+\mathrm{SC}$.

\subsection{Robustness}

Robustness refers to the ability of watermarking scheme to withstand against intentional or unintentional attacks, that may be applied on watermarked images either to remove or to destroy the hidden information [7], [21]. Robustness is measured using normalized correlation [6] shown in (24). In order to check the robustness of proposed schemes different attacks like rotation (RO), translation (TR), $\mathrm{x}$-shearing (XSH), y-shearing (YSH), scaling (SC), cropping (CR), affine transformation (AFT), Gaussian noise (GN), salt \& pepper noise 


\begin{tabular}{|c|c|c|c|c|c|c|}
\hline \multicolumn{2}{|c|}{ Attacks } & \multicolumn{5}{|c|}{ Constant Scaling Factor $(\beta)$} \\
\hline Attack & Parameters & 0.1 & 0.3 & 0.5 & 0.7 & 0.9 \\
\hline \multirow{3}{*}{ Rotation } & $\theta=45$ & 0.8031 & 0.7283 & 0.8229 & 0.8211 & 0.7286 \\
\hline & $\theta=60$ & 0.8258 & 0.8279 & 0.8103 & 0.8098 & 0.8112 \\
\hline & $\theta=125$ & 0.8272 & 0.8064 & 0.8068 & 0.8064 & 0.8066 \\
\hline \multirow{2}{*}{ Translation } & displacement $60 \%$ & 0.8142 & 0.8135 & 0.8135 & 0.8596 & 0.8117 \\
\hline & displacement $120 \%$ & 0.8089 & 0.7444 & 0.7444 & 0.8084 & 0.7441 \\
\hline \multirow{2}{*}{ X-Shearing } & Shearing Factor $=0.4$ & 0.7998 & 0.7987 & 0.8466 & 0.7982 & 0.8466 \\
\hline & Shearing Factor $=-0.5$ & 0.7989 & 0.7996 & 0.8466 & 0.8488 & 0.7980 \\
\hline \multirow{2}{*}{ Y-Shearing } & Shearing Factor $=-0.4$ & 0.8488 & 0.8322 & 0.7873 & 0.7899 & 0.8455 \\
\hline & Shearing Factor $=0.5$ & 0.7715 & 0.8413 & 0.8418 & 0.7722 & 0.7717 \\
\hline Affine Transformation & Transform Factor $=0.5$ & 0.7563 & 0.7551 & 0.7558 & 0.8188 & 0.7556 \\
\hline \multirow{2}{*}{ Scaling } & 3times & 0.8712 & 0.8695 & 0.8693 & 0.8682 & 0.8693 \\
\hline & 0.5 times & 0.8792 & 0.8800 & 0.8796 & 0.8787 & 0.8783 \\
\hline \multirow{2}{*}{ Cropping } & $10 \%$ & 0.8676 & 0.8682 & 0.8678 & 0.8676 & 0.8674 \\
\hline & $25 \%$ & 0.8646 & 0.8646 & 0.8648 & 0.8641 & 0.8646 \\
\hline \multirow{2}{*}{ Gaussian Noise } & $\mu=0.4, \sigma^{2}=.01$ & 0.8654 & 0.8635 & 0.8659 & 0.8678 & 0.8643 \\
\hline & $\mu=0.5, \sigma^{2}=0.5$ & 0.8641 & 0.8563 & 0.8587 & 0.8663 & 0.8700 \\
\hline \multirow{2}{*}{ Salt \& Pepper Noise } & Density $=0.1$ & 0.8652 & 0.8633 & 0.8704 & 0.8654 & 0.8691 \\
\hline & Density $=0.5$ & 0.8609 & 0.8650 & 0.8704 & 0.8652 & 0.8609 \\
\hline \multirow{2}{*}{ Speckle Noise } & Density $=0.1$ & 0.8725 & 0.8689 & 0.8667 & 0.8689 & 0.8732 \\
\hline & Density $=0.5$ & 0.8695 & 0.8738 & 0.8585 & 0.8567 & 0.8598 \\
\hline \multirow{2}{*}{ Blurring } & Motion Blurring & 0.8678 & 0.8687 & 0.8689 & 0.8700 & 0.8691 \\
\hline & Simple Blurring & 0.8719 & 0.8721 & 0.8715 & 0.8706 & 0.8704 \\
\hline \multirow[b]{2}{*}{ Average Filtering } & $5 \times 5$ & 0.8836 & 0.8834 & 0.8834 & 0.8830 & 0.8836 \\
\hline & $7 \times 7$ & 0.8757 & 0.8747 & 0.8730 & 0.8727 & 0.8738 \\
\hline Histogram Equalization & & 0.8661 & 0.8667 & 0.8665 & 0.8665 & 0.8663 \\
\hline JPEG Compression & Quality Factor $=50$ & 0.8611 & 0.8624 & 0.8617 & 0.8617 & 0.8600 \\
\hline \multirow{5}{*}{ Combined Watermarking Attacks } & JPEG Comp+Filtering $(5 \times 5)$ & 0.8745 & 0.8753 & 0.8762 & 0.8751 & 0.8747 \\
\hline & JPEG Comp + Gaussian Noise $(0.5)$ & 0.8643 & 0.8730 & 0.8680 & 0.8723 & 0.8736 \\
\hline & JPEG Comp + Scaling (Half) & 0.8811 & 0.8813 & 0.8804 & 0.8811 & 0.8800 \\
\hline & Translation+Shearing & 0.8429 & 0.8493 & 0.8521 & 0.8623 & 0.8670 \\
\hline & Rotation $\left(45^{\circ}\right)+$ scaling & 0.8133 & 0.8084 & 0.7479 & 0.7471 & 0.8270 \\
\hline
\end{tabular}

Tab. 6. NC For Different Attacks (Proposed Scheme 1).

$(\mathrm{S} \& \mathrm{P})$, speckle noise $(\mathrm{SN})$, motion blurring $(\mathrm{MB})$, simple blurring (SB), average filtering (AF), histogram equalization (HE) and JPEG compression (JQ) were applied on watermarked image. In addition to conventional watermarking attacks, combined attacks are formed by combining two or more conventional attacks. For instance, watermark is tried to be extracted form a watermarked images that has been subjected to JPEG compression and filtering attack. In this way five additional attacks - JPEG compression plus filtering (JQ+AF), JPEG compression plus Gaussian noise (JQ+GN), JPEG compression plus scaling (JQ+SC), translation plus $\mathrm{x}$ and y shearing $(\mathrm{TR}+\mathrm{SH})$, and rotation plus scaling $(\mathrm{RO}+\mathrm{SC})$ - are also used to check robustness of proposed schemes

$$
N C=\frac{\sum_{p=1}^{P} \sum_{q=1}^{Q}(W(p, q) \times \hat{W}(p, q))}{\sqrt{\sum_{p=1}^{P} \sum_{q=1}^{Q} W^{2}(p, q)} \times \sqrt{\sum_{p=1}^{P} \sum_{q=1}^{Q} \hat{W}^{2}(p, q)}} .
$$

For qualitative assessment of extracted watermarks after applying above mentioned attacks on watermarked image, Fig. 7 can be referred. It can be seen that in all cases the extracted watermarks are clearly visible hence can be used to prove ownership. The performance of proposed scheme 1 in terms of robustness for different scaling factors is shown in Tab. 6. In general with increasing scaling factor the robustness is increased [3], whereas, the change in $\mathrm{NC}$ values shown in Tab. 6 , is random and that is due to the adaptive scaling factor $\left(\alpha_{M L}\right)$.

The comparison of proposed schemes in terms of NC with existing technique [3], [6], [13] and [14] is shown in Tab. 7.

Robustness of watermarking techniques are also measured using Bit Error Rate (BER) [3], shown in (25). The comparision of proposed schemes in terms of BER with [3], [6], [13] and [14] is shown in Tab. 8. From Tab. 7 and Tab. 8, it is clear that the proposed schemes give better results in terms of robustness as well.

$$
B E R=\frac{\text { Number of wrong bits extracted }}{\text { Total number of bits embedded }} .
$$

The extracted watermarks shown in Fig. 4 are for proposed scheme 1 , however, the quality of watermarks obtained using proposed scheme 2 is also good. This can be seen from Tab. 7 that there is not significant difference between NC 


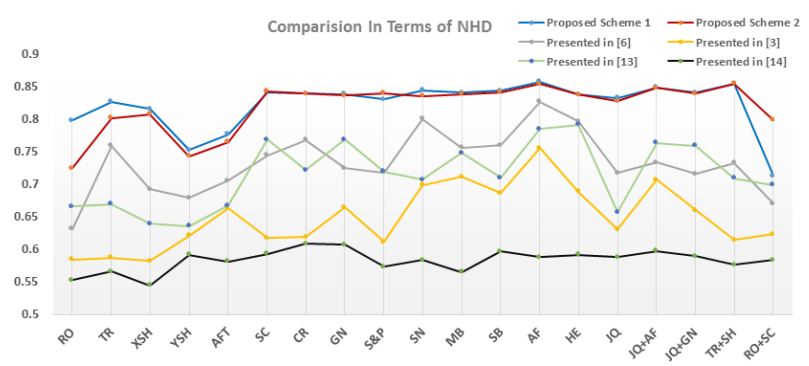

(a)

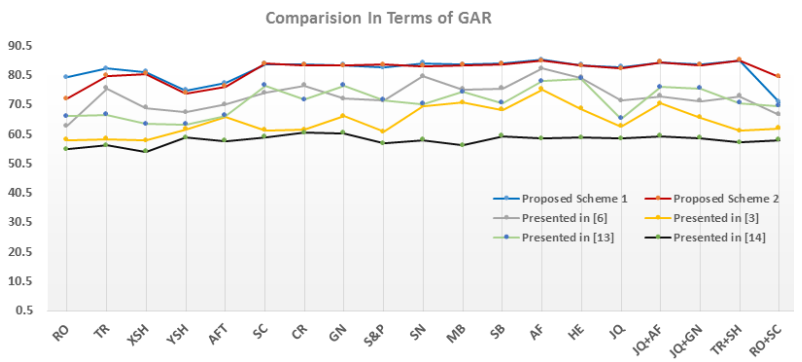

(b)

Fig. 5. Comparison of proposed schemes with existing techniques (a). Comparison in terms of NHD (b). Comparison in terms of GAR.

\begin{tabular}{|c|c|c|c|c|c|c|}
\hline \multirow{3}{*}{${ }^{7} c_{\sigma_{0 / T_{s}}}$} & \multicolumn{6}{|c|}{ For Constant Scaling Factor $\beta=\mathbf{0 . 5}$} \\
\hline & \multicolumn{2}{|c|}{ Proposed Schemes } & \multicolumn{4}{|c|}{ Presented in } \\
\hline & 1 & 2 & [6] & [3] & {$[13]$} & [14] \\
\hline $\mathrm{RO}$ & $\overline{82}$ & 80 & .5894 & .5377 & 0.6274 & 0.52 \\
\hline$\overline{\mathrm{TR}}$ & 135 & & 5946 & 5182 & 0.60 & 0.55 \\
\hline $\mathrm{XSH}$ & 466 & 83 & .6148 & 0.5002 & 0.6633 & 0.544 \\
\hline$\overline{\mathrm{YSH}}$ & & & 2 & 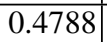 & 0.6779 & 0.62 \\
\hline AFT & 7558 & 0.8060 & 0.6376 & 0.4885 & 0.7029 & 0.538 \\
\hline $\mathrm{SC}$ & & & 4 & 6 & 0.7 & 0.5 \\
\hline $\mathrm{CR}$ & 8648 & 0.8570 & 0.6825 & 0.6322 & 0.7370 & 0.621 \\
\hline GN & & & 38 & 0.5797 & 0.7896 & 0.58 \\
\hline $\mathrm{S} \& \mathrm{P}$ & 3704 & 0.8656 & 0.7406 & 0.5270 & 0.7420 & 0.543 \\
\hline SN & 67 & & 04 & 0.6247 & 0.7375 & 0.55 \\
\hline MB & 689 & 16 & 0.7859 & 0.6355 & 0.7772 & 0.587 \\
\hline SB & 15 & 65 & 7824 & 0.6555 & 0.7312 & 0.55 \\
\hline$\overline{\mathrm{AF}}$ & 834 & & 7811 & 0.6577 & 0.7417 & 0.556 \\
\hline $\mathrm{HE}$ & 665 & 0 & 539 & 0.5576 & 0.7493 & 0.560 \\
\hline JQ & 0.8617 & 0.8039 & 0.7488 & 0.5538 & 0.6861 & 0.562 \\
\hline $\mathrm{JQ}+\mathrm{AF}$ & 0.8762 & 0.8753 & 0.7568 & 0.5234 & 0.7882 & 0.534 \\
\hline JQ+GN & 0.8680 & 0.8643 & 0.7375 & 0.5769 & 0.7822 & 0.587 \\
\hline $\mathrm{JQ}+\mathrm{SC}$ & 0.8804 & 0.8813 & 0.7554 & 0.5305 & 0.7316 & 0.542 \\
\hline $\mathrm{TR}+\mathrm{SH}$ & 0.8521 & 0.8475 & 0.7058 & 0.5028 & 0.7238 & 0.512 \\
\hline $\mathrm{RO}+\mathrm{SC}$ & 0.7479 & 0.8308 & 0.6973 & 0.5028 & 0.7276 & 0.534 \\
\hline
\end{tabular}

Tab. 7. NC for different techniques.

values of both proposed schemes. The recognizable watermarks can be extracted as long as the distorted watermarked image are visually identifiable.

Besides NC and BER, there are other ways to calculate the credibility of extracted watermarks quantitatively. For instance, Normalized Hamming Distance (NHD) [6] shown in 26 , where, $w$ and $\hat{w}$ represent embedded and extracted watermark respectively, can be used to calculate the similarity (or difference) between embedded and extracted watermarks.

$$
H_{\mathrm{D}}=\frac{\sum_{1=1}^{m} \sum_{j=1}^{n} h_{w \hat{w}}(i, j)}{m \times n}
$$

where

$$
h_{w \hat{w}}(i, j)= \begin{cases}1, & \text { if } w(i, j)=\hat{w}(i, j), \\ 0, & \text { otherwise. }\end{cases}
$$

\begin{tabular}{|c|c|c|c|c|c|c|}
\hline \multirow{3}{*}{${ }^{7}{ }^{8 / 2 / s}$} & \multicolumn{6}{|c|}{ For Constant Scaling Factor $\beta=\mathbf{0 . 5}$} \\
\hline & \multicolumn{2}{|c|}{ Proposed Schemes } & \multicolumn{4}{|c|}{ Presented in } \\
\hline & 1 & 2 & [6] & [3] & [13] & [14] \\
\hline RO & 0.2107 & 0.2256 & 0.2360 & 0.3679 & 0.3153 & 0.3791 \\
\hline TR & 0.1792 & 0.1736 & 0.1907 & 0.2933 & 0.2510 & 0.2717 \\
\hline $\mathrm{XSH}$ & 0.1848 & 0.1892 & 0.2268 & 0.2951 & 0.2225 & 0.2712 \\
\hline YSH & 0.1902 & 0.1819 & 0.2965 & 0.2984 & 0.2108 & 0.2276 \\
\hline AFT & 0.2798 & 0.2231 & 0.2577 & 0.3570 & 0.2481 & 0.3239 \\
\hline $\mathrm{SC}$ & 0.1477 & 0.1580 & 0.1939 & 0.2738 & 0.2200 & 0.3231 \\
\hline $\mathrm{CR}$ & 0.1611 & 0.1692 & 0.1920 & 0.2799 & 0.2401 & 0.2847 \\
\hline GN & 0.1672 & 0.1677 & 0.1829 & 0.2801 & 0.2056 & 0.2788 \\
\hline S\&P & 0.1582 & 0.1597 & 0.1454 & 0.2798 & 0.1987 & 0.2713 \\
\hline SN & 0.1660 & 0.1531 & 0.1825 & 0.2857 & 0.2420 & 0.3207 \\
\hline MB & 0.1599 & 0.1531 & 0.1833 & 0.2769 & 0.2264 & 0.2996 \\
\hline SB & 0.1570 & 0.1587 & 0.1761 & 0.2758 & 0.2472 & 0.3271 \\
\hline $\mathrm{AF}$ & 0.1433 & 0.1477 & 0.1652 & 0.2601 & 0.2306 & 0.3076 \\
\hline $\mathrm{HE}$ & 0.1626 & 0.2517 & 0.1130 & 0.2769 & 0.2061 & 0.2757 \\
\hline JQ & 0.1680 & 0.1616 & 0.2064 & 0.2760 & 0.2228 & 0.2719 \\
\hline $\mathrm{JQ}+\mathrm{AF}$ & 0.1516 & 0.1526 & 0.1985 & 0.2864 & 0.2318 & 0.2945 \\
\hline $\mathrm{JQ}+\mathrm{GN}$ & 0.1609 & 0.1650 & 0.2053 & 0.2356 & 0.2514 & 0.2718 \\
\hline $\mathrm{JQ}+\mathrm{SC}$ & 0.1467 & 0.1458 & 0.1856 & 0.2281 & 0.2346 & 0.2815 \\
\hline $\mathrm{TR}+\mathrm{SH}$ & 0.1656 & 0.1715 & 0.1983 & 0.2459 & 0.2287 & 0.2568 \\
\hline $\mathrm{RO}+\mathrm{SC}$ & 0.1876 & 0.2021 & 0.2598 & 0.2983 & 0.2795 & 0.3143 \\
\hline
\end{tabular}

Tab. 8. BER for different techniques.

Similarly, Global Authentication Rate (GAR) [22] shown in (27) is also used to check the quality of extracted watermark. It should be noted that GAR and NHD are almost same just different form of representations

$$
\varrho^{\mathrm{GAR}}=\left(1-\frac{1}{m \times n} \sum_{i=1}^{m} \sum_{j=1}^{n}(w(i, j) \oplus \hat{w}(i, j))\right) \times 100 \%
$$

The performance of proposed schemes is examined using both NHD and GAR in terms of robustness and also compared with other watermark techniques.

The comparision of proposed and existing watermarking schemes in terms of NHD is shown in Fig. 5(a) and in terms of GAR in Fig. 5(b). Both figures clearly demonstrate the improvement of proposed scheme over existing watermarking techniques. 


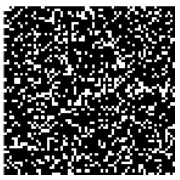

(a)

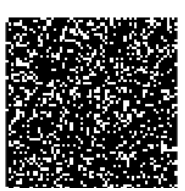

(e)

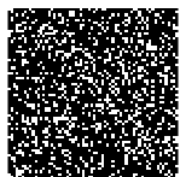

(i)

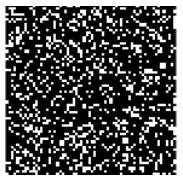

(b)

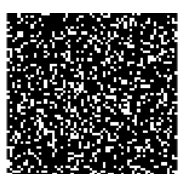

(f)

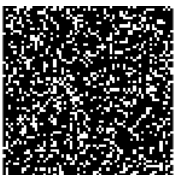

(j)

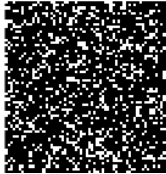

(c)

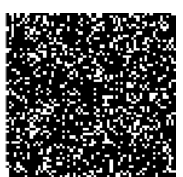

(g)

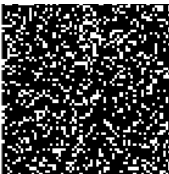

(k)

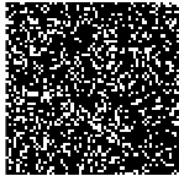

(d)

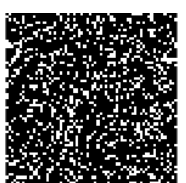

(h)

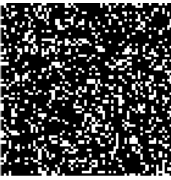

(1)
Fig. 6. Extracted watermarks with fake keys (proposed scheme 1).

\subsection{Security}

Security refers to the resistance against false positive or true negative extraction of watermark [5]. In designing the proposed schemes security is given high importance and it was ensured to nullify the chances of false positive or true negative detection or extraction of hidden information. To test the security ample number of fake keys were used to extract the watermark neither true nor false watermark was extracted. In Fig. 6 the extracted watermarks with false keys are shown. It is evident that no watermark was extracted.

\subsection{Capacity}

Capacity refers to the amount of data that can be embedded into the host image without degrading the quality of watermarked image. The capacity of proposed schemes is $(64 \times 64 \times 3=12288)$, since watermark of size $64 \times 64$, and all three components $(U, S$ and $V)$ of SVD decomposition are used. This is considered to be good capacity for a watermarking scheme. The capacities of proposed schemes are 3 times more than the capacities of [3] and [6].

\section{Conclusion}

In this paper, two watermarking schemes are proposed. In order to achieve four conflicting requirements for a good watermarking scheme, HVS, FIS, PCA and SVD are used together. The perceptual quality is improved by using PCA to decorrelate the three channels of color image, afterwards HVS and FIS are used to find adaptive scaling factor so that the amount of information embedded is subject to the acceptability of host image. For instance, the areas which are less prone to modifications are modified to lesser extent as compared to areas which are open to changes. In this way imperceptibility is further improved, and can be seen from results that the imperceptibility of proposed scheme 1 is much better than proposed scheme 2 (HVS and FIS are not employed in proposed scheme 2, otherwise it is same as proposed scheme 1). To achieve robustness, SVD is used, as changes in singular values does not change the image and vice versa. Whereas to obtain security, based on correlation certain elements from SVD components are selected for modification, and then those locations served as key at the time of watermark extraction. In this way not only security and capacity are achieved but this method also helped to improve the imperceptibility as suggested by results. The proposed schemes are compared with state-of-the-art watermarking techniques and obtained better results.

\section{References}

[1] TSUI, K. T., ZHANG, P. X., ANDROUTSOS, D. Color image watermarking using multidimensional Fourier transforms. IEEE Transactions on Information Forensics and Security, 2008, vol. 3, no. 1, p. 1556-6013. DOI: 10.1109/TIFS.2007.916275

[2] CHOU, H. C., LIU, C. K. A perceptually tuned watermarking scheme for color images. IEEE Trans. on Image Processing, 2010, vol. 19 , no. 11, p. 2966-2982. DOI: 10.1109/TIP.2010.2052261

[3] CEDILLO-HERNANDEZ, M. CEDIlLO-HERNANDEZ, A GARCIA-UGALDE, F., et. al. Copyright protection of color imaging using robust-encoded watermarking. Radioengineering, 2015, vol. 24 , no. 1, p. 240-251. DOI: 10.13164/re.2015.0240

[4] SU, Q., NIU, Y., ZOU, H., et el. A blind dual color image watermarking based on singular value decomposition. International Journal of Applied Mathematics and Computation, 2013, vol. 219, no. 16, p. 8455-8466. DOI: 10.1016/j.amc.2013.03.013

[5] MAKBOOL, N. M., KHOO, E. B. A new robust and secure digital image watermarking scheme based on the integer wavelet transform and singular value decomposition. International Journal of Digital Signal Processing, 2014, vol. 33, p. 134-147. DOI: $10.1016 /$ j.dsp.2014.06.012

[6] PRATHAP, I., NATARAJAN, V., ANITHA, R. Hybrid robust watermarking for color images. International Journal of Computers \& Electrical Engineering, 2014, vol. 14, no. 3, p. 920-930. DOI: 10.1016/j.compeleceng.2014.01.006

[7] GHAFOOR, A., IMRAN, M. A non-blind color image watermarking scheme resistant against geometric attacks. Radioengineering, 2012, vol. 21 , no. 4 , p. 1246-1251. ISSN: $1805-9600$

[8] BAISA, L., GUNJAL, MALI, N. S. Comparative performance analysis of DWT-SVD based color image watermarking technique in YUV, RGB and YIQ color spaces. International Journal of Computer Theory and Engineering, 2011, vol. 3, no. 6, p. 714-717.

[9] SUN, X., BO, S. A blind digital watermarking for color medical images based on PCA. In Proceedings of the IEEE International Conference on Wireless Communications, Networking, and Information Security (WCNIS). Beijing (China), 2010, p. 421-427. DOI: 10.1109/WCINS.2010.5541812

[10] LIU, R., TAN, T. An SVD based watermarking scheme for protecting rightful information. IEEE Transactions on Multimedia, 2002, vol. 4 , no. 1 , p. 121-128. DOI: $10.1109 / 6046.985560$

[11] LAI, C. C., TAI, C. C. Digital image watermarking using discrete wavelet transform and singular value decomposition. IEEE Transactions on Instrumentation and Measurement, 2010, vol. 59, no. 11, p. 3060-3063. DOI: 10.1109/TIM.2010.2066770 
[12] LAI, C. C. A digital watermarking scheme based on singular value decomposition and tiny genetic algorithm. International Journal of Digital Signal Processing, 2011, vol. 21, no. 4, p. 522-527. DOI: 10.1016/j.dsp.2011.01.017

[13] FAZLI, S., MOEINIi, M. A robust image watermarking method based on DWT, DCT, and SVD using a new technique for correction of main geometric attacks. International Journal of Optik, 2016, vol. 127, no. 2, p. 964-972. DOI: 10.1016/j.ijleo.2015.09.205

[14] ROY, A., MAITI, K. A., GHOSH, K. A perception based color image adaptive watermarking scheme in $\mathrm{YCbCr}$ space. In Proceedings of the IEEE International Conference on Signal Processing and Integrated Networks. 2015, p. 537-543. DOI: 10.1109/SPIN.2015.7095399

[15] QI, H., ZHENG, D., ZHAO, J. Human visual system based adaptive digital image watermarking. International Journal of Signal Processing, 2008, vol. 88, no. 1, p. 174-188. DOI: 10.1016/j.sigpro.2007.07.020

[16] GOU, J. M., PRASETYO, H. A novel gray-scale image watermarking using hybrid fuzzy-BPN architecture. International Journal of Visual Communication and Image Representation, 2014, vol. 25, no. 5, p. 1149-1163. DOI: 10.1016/j.eij.2015.01.002

[17] WANG, X., L. A Course in Fuzzy Systems and Control. 1st ed., USA: Prentice Hall, 1997. ISBN: 978-0135408827

[18] JOLlifFE, T. I. Principal Component Analysis. 2nd ed., USA: Springer, 2002. ISBN: 978-0-387-22440-4

[19] WANG, Z. BOVIK, C. A., SHEIKH, R. H., et al. Image quality assessment: from error visibility to structural similarity. IEEE Transactions on Image Processing, 2004, vol. 13, no. 4, p. 600-612. DOI: 10.1109/TIP.2003.819861

[20] SHEIKH, R. H., BOVIK, C. A. T. S. Image information and visual quality. IEEE Transactions on Image Processing, 2006, vol. 15, no. 2 , p. 430-444. DOI: 10.1109/TIP.2005.859378

[21] LEVICKY. D., FORIS, P. Human visual system models in digital image watermarking. Radioengineering, 2004, vol. 13, no. 4, p. 28-43. ISSN: $1805-9600$

[22] LIE, N. W., LIN, S. G., CHENG, L. S. Dual protection of JPEG images based on informed embedding and two-stage watermark extraction techniques. IEEE Transactions on Information Forensics and Security, 2006, vol. 1, no. 3, p. 330-341. DOI: 10.1109/TIFS.2006.879297

\section{About the Authors...}

Muhammad IMRAN received the B.Eng. degree in electronic engineering from Mehran University of Engineering and Technology (MUET), Jamshoro, Pakistan, in 2007 and the M. Sci. degree in electrical engineering from National University of Sciences and Technology (NUST), Islamabad, Pakistan, in 2012. He is currently pursuing the PhD degree in electrical engineering at the Florida State University. He is a research assistant in Image Processing and Communication Research Laboratory. His research interests are in digital image processing, digital image watermarking and steganography.

Bruce A. HARVEY received the B.E.E. (Co-op, Highest Honor) from Auburn University in 1984, the M.S.E.E. from the University of Alabama in Huntsville in 1987 and the Ph.D. in Electrical Engineering from Georgia Institute of Technology in 1991. He was a Research Engineer I at the Georgia Tech Research Institute (GTRI) from 1984-1986 and a Lead Engineer at Phase IV Systems, Inc. from 1986-1988. From 1991-1997 he was a Research Engineer in the Communications Division of GTRI. In 1997 he joined the Department of Electrical and Computer Engineering at the FAMU-FSU College of Engineering in Tallahassee, Florida, and currently holds the rank of Associate Professor. His current fields of interest include lightning surge suppression, wireless communication, error control coding, wireless networks, modulation techniques, digital image processing, digital image watermarking, and modeling and analysis. Dr. Harvey is a member of the IEEE Communications and Education Societies. 